\title{
La fin de l'en-dehors : les nouvelles constructions discursives de l'ennemi intérieur en Californie
}

Ayse Ceyhan

\section{(2) OpenEdition}

1 Journals

\section{Édition électronique}

URL : http://journals.openedition.org/conflits/569

DOI : $10.4000 /$ conflits.569

ISSN : $1777-5345$

Éditeur :

CCLS - Centre d'études sur les conflits lilberté et sécurité, L'Harmattan

Édition imprimée

Date de publication : 1 septembre 2001

ISBN : 2-7475-1118-9

ISSN : 1157-996X

\section{Référence électronique}

Ayse Ceyhan, «La fin de l'en-dehors : les nouvelles constructions discursives de l'ennemi intérieur en Californie », Cultures \& Conflits [En ligne], 43 | automne 2001, mis en ligne le 28 février 2003, consulté le 30 mars 2021. URL : http://journals.openedition.org/conflits/569 ; DOl : https://doi.org/10.4000/ conflits.569

Ce document a été généré automatiquement le 30 mars 2021.

Creative Commons License 


\title{
La fin de l'en-dehors : les nouvelles constructions discursives de l'ennemi intérieur en Californie
}

\author{
Ayse Ceyhan
}

Quels sont les liens discursifs entre immigration et ennemi intérieur dans un pays réputé pour être un pays d'immigration et de tolérance ? Il est vrai que quand à partir des années quatre-vingts, les pays européens ont vu se développer un discours de peur et de danger portant sur les immigrés originaires des pays dits du Sud, tout portait à croire à leur singularité. Or, presque au même moment se développaient aux Etats-Unis des discours de "démonisation » de l'immigration, accompagnés de la mise en place d'une série de technologies de surveillance et de contrôle aux frontières et à l'intérieur du territoire.

Paradoxalement, c'est en Californie - l'Etat le plus riche et le plus multiculturel de l'Union où un tiers de la population est d'origine hispanique et un dixième asiatique que la transformation de l'immigration en problème politique et en enjeu sécuritaire a été la plus perceptible. Ainsi, dans le contexte d'un référendum local organisé le 8 novembre 1994, les électeurs californiens ont adopté avec une majorité de 59,1\%, la Proposition 187 qui refusait aux clandestins le bénéfice des droits sociaux (le Welfare) et interdisait à leurs enfants l'accès à l'enseignement public en prévoyant leur expulsion vers leur pays d'origine. Cette loi allait même plus loin puisqu'elle obligeait les enseignants, les médecins et les assistantes sociales à dénoncer aux autorités policières les personnes suspectes, facilement identifiables au faciès et à l'accent.

L'adoption de la Proposition 187 a non seulement provoqué la transformation de l'immigration en un problème politique et sécuritaire, mais a permis de déplacer l'image de l'ennemi du «dehors" vers le «dedans » en mettant en place tout un processus discursif d'énonciation, de désignation, de dénonciation et de dramatisation. Dorénavant, la figure de l'ennemi n'était plus représentée par le communisme qui pendant longtemps avait incarné la menace externe, mais s'était transposée à l'intérieur où elle avait pris une forme plus complexe et plus diffuse. Ainsi, non définie 
clairement, elle pouvait prendre plusieurs visages dont celui de l'immigré venu du Sud considéré comme potentiellement nuisible à la sécurité identitaire et à la sécurité intérieure du pays.

En attribuant la responsabilité des problèmes économiques et sociaux aux immigrés en situation illégale et en transformant l'immigration en général en un problème politique et sécuritaire, la Proposition 187 présentait les immigrés comme la cause de tous les maux du moment, allant des problèmes économiques comme la crise de l'Etatprovidence à la violence urbaine (plus particulièrement suite aux événements de South Central de 1992) en passant par la criminalité, le trafic de drogue, les incivilités et même la décomposition de la cellule familiale traditionnelle. En nous référant à Murray Edelman ${ }^{1}$, nous dirons que l'« ennemi » est un «fourre-tout» qui s'inscrit dans un processus de construction équivoque d'un problème politique dont la principale fonction est la négation des problèmes structurels comme le chômage qui préoccupent la société. La polarisation sur un "ennemi » inoffensif permet de transformer une situation donnée en un problème dont la construction passe par le langage qui matérialise l'ennemi même si celui-ci est volontairement flou. Dans cette construction, les problèmes politiques ne sont pas définis clairement et se caractérisent par l'ambiguïté que créent leurs dispositifs narratifs et par la non-résolution des controverses soulevées par leurs significations. Ils justifient les solutions comme l'adoption des politiques sécuritaires et constituent des autorités (experts, spécialistes, professionnels, conseillers du prince, etc). Par ailleurs, tributaires de luttes politiques, les problèmes créent des bénéfices politiques pour certains acteurs et augmentent l'influence du groupe bénéficiaire. Enfin ils influencent les agendas politiques et constituent des opinions publiques².

Interprétée à la hâte comme une politique symbolique sans grand effet réel, la Proposition 187, même si elle a été déclarée anticonstitutionnelle ${ }^{3}$ par le tribunal fédéral, n'est pourtant pas un épiphénomène mais plutôt un événement révélateur ${ }^{4}$. En effet, par les débats qu'elle a suscités, les mesures qu'elle a permis d'adopter, les discours qu'elle a produits, les pratiques qu'elle a générées, elle a permis de saisir les modifications importantes survenues dans la conception américaine de l'immigration et de la menace depuis les années 1980. Plus spécifiquement, elle a permis de comprendre l'introduction dans les années 1990 d'une nouvelle figure de l'ennemi (l'immigré) dans les espaces discursifs politiques et sécuritaires américains.

Dans cet article, nous analyserons l'historicité de l'idée de l'ennemi intérieur (ou l'ennemi de l'intérieur) en montrant son lien avec les étrangers arrivés de frâiche date sur le territoire américain. Ensuite, nous analyserons comment dans le contexte de l'après-Guerre Froide, la Proposition 187 a transformé l'immigration en un problème politique et en la nouvelle figure de l'ennemi. Pour cela nous verrons comment elle s'est inscrite dans un dispositif complexe et comment elle a crée des effets de vérité 5 . Nous analyserons ses régimes d'énoncé et ses procédés argumentatifs. Considérant que les dispositifs ne sont pas homogènes, nous verrons les discours de résistance qu'elle a générés. De même, nous nous interrogerons sur les jeux politiques et bureaucratiques qui ont permis la construction de cette nouvelle figure de l'ennemi intérieur qu'est l'immigré à travers un processus de problématisation. 


\section{A la recherche d'une nouvelle figure de l'ennemi}

Afin de mieux saisir les caractéristiques de la Proposition 187, il convient de la situer dans son contexte historique et socio-politique. Rappelons qu'elle a émergé après la chute du Mur de Berlin, dans un moment de restructuration économique face à la perte de milliers d'emplois, conséquence de la réduction des effectifs de la défense et des industries qui lui étaient liées. Ces industries étaient principalement basées en Californie où l'armée était le principal pourvoyeur. De même, la Proposition 187 a été forgée dans un contexte où la menace externe (le communisme) ne structurait plus la politique interne et externe. En effet, pendant la Guerre Froide, le communisme - et tout ce qui depuis les films hollywoodiens jusqu'aux mouvements de libération, pouvait être identifié comme communiste - constituait la figure de l'ennemi à partir de laquelle se construisait le discours de la menace. C'était une menace unifiée et identifiable qui engendrait une figure de l'ennemi pouvant s'articuler avec la notion de cinquième colonne c'est-à-dire ceux qui comme "les intellectuels déviants » étaient accusés de soutenir la cause de l'ennemi situé à l'extérieur'.

Mais dans le contexte international actuel, les phénomènes de globalisation, de transnationalisation, de déterritorialisation et de suppression des frontières remettent progressivement en cause la différence absolue entre l'interne et l'externe, le national et l'international, le public et le privé. Ce processus s'accompagne de l'abandon de la dialectique du dehors et du dedans, et l'en-dehors au sens du réalisme politique et militaire n'a plus de sens. Ainsi, comme le remarque Michael Hardt ${ }^{7}$, dans le contexte de l'interpénétration croissante de l'interne et de l'externe, la puissance souveraine n'est plus opposée à son Autre, elle n'est plus confrontée à son dehors, il n'y a plus de dehors pour borner le lieu de la souveraineté. Aussi « de nos jours, il est de plus en plus difficile pour les idéologues des Etats-Unis de nommer l'ennemi ou plutôt il semble qu'il y ait partout des ennemis mineurs et insaisissables ${ }^{8}$. Cette situation affecte non seulement la structuration de la politique mais aussi la (re)définition de l'identité américaine. Car, comme le rappelle David Campbell, dans un pays comme les EtatsUnis, où être américain n'est pas clairement défini, le discours sur la menace joue un rôle fondamental dans la formation de l'identité politique ainsi que dans les pratiques d'exclusion et d'inclusion'.

Il nous faudra rappeler enfin que la recherche de nouvelles figures de l'ennemi a commencé dès les années 1980, bien avant la suppression de la menace communiste. Les politiciens et les agences de sécurité ont évoqué une multiplication de dangers venant du terrorisme international, de la mafia, du trafic de drogue. Ils ont même évoqué « l'ennemi japonais » envahissant Hollywood, l'« ennemi coréen » envahissant l'industrie automobile, mais ces figures ont vite été oubliées. Elles constituaient peutêtre un danger sectoriel mais non pas une menace susceptible de structurer une politique et un discours cohérents. Dans ce nouveau contexte, il fallait trouver un autre visage à l'ennemi, un visage qui a trait aux nouvelles préoccupations de sécurité, celles qui reposent sur les questions de culture, d'identité, d'harmonie de la nation, d'équilibre démographique et sur l'ordre public. En effet, avec l'élargissement du spectre de la sécurité à de nouveaux secteurs et objets ${ }^{10}$, on se recentrait davantage sur la sécurité à l'intérieur du pays que sur la menace externe ${ }^{11}$. Ainsi, il ne s'agissait plus seulement de contrôler le passage des frontières, mais de surveiller les possibles 
menaces culturelles, identitaires, démographiques, écologiques, etc. Peut-on dire que ces préoccupations ont fait glisser la notion d'ennemi de l'extérieur vers l'intérieur?

\section{L'idée de l'ennemi intérieur}

A l'heure du brouillage des frontières entre l'interne et l'externe, parler de l'ennemi intérieur peut paraître obsolète si l'on prend celui-ci dans un rapport binaire comme opposé à l'externe. En effet, dans la tradition du réalisme politique, l'ennemi intérieur est celui qui se lie avec l'ennemi extérieur. Et on a souvent coutume de le désigner par l'expression "cinquième colonne ",phénomène politico-social où la trahison est le fait de la manipulation des esprits par l'ennemi de l'intérieur magistralement téléguidé par celui de l'extérieur. Mais selon Alexandre Koyré12 ${ }^{12}$ l'ennemi intérieur ne dépend pas seulement du rapport à l'externe et ne se réduit pas uniquement à la "cinquième colonne $»^{13}$, mais résulte également de la perte par l'Etat national de sa structure monolithique qui lui avait permis d'unifier, de lier et d'absorber les populations en son sein. Ainsi selon lui « on aurait tort d'appliquer le terme 'cinquième colonne' d'une manière indiscriminée à toutes les espèces différentes $d$ "ennemis intérieurs' dont l'activité constitue un danger actuel ou potentiel pour l'Etat ${ }^{14}$. Il y a plusieurs espèces d'ennemis intérieurs dont l'existence implique et indique pour Koyré « la présence au sein de la Cité de groupes non-intégrés, non embrassés par le lien social; de groupes qui refusent de s'identifier avec le Tout de la Cité, ainsi que de se solidariser - dans ce Tout - avec les autres groupes qui les composent et le construisent; de groupes qui s'isolent ou qui se trouvent isolés - dans ce Tout; qui s'opposent à ce Tout; qui l'opposition s'intensifiant et s'exaspérant, passent de l'opposition à l'hostilité, de l'hostilité à la haine ; le cas échéant la lutte sourde se transformera en lutte ouverte, la sédition fera son entrée dans l'Etat $»^{15}$. Ce sont «les 'Autres', les 'barbares', ceux qui n'ont pas la même origine, qui ne parlent pas ou parlent mal la langue nationale, qui ne partagent pas les souvenirs historiques, qui ne participent pas aux fêtes traditionnelles de la nation et qui par hasard seulement, hasard de conquête, de migration, de liaison dynastique - font partie de l'Etat $\aleph^{16}$. Ces citations permettent de voir comment l'ennemi intérieur est présenté comme étant lié à l'Autre, à l'étranger, au différent et à l'exclu. Si nous transposons cette construction au cas de la Californie, elle pourrait nous permettre de voir comment l'idée de l'ennemi intérieur fonctionne par rapport aux étrangers originaires des pays du Suden général, et aux Mexicains en particulier. Elle indique comment virtuellement l'ennemi intérieur est lié aux questions d'assimilation, de langue, de multiculturalisme et comment il s'articule avec les idées de l'harmonie de la nation et de la protection des valeurs américaines.

Par ailleurs, si nous nous situons dans la perspective d'Edelman, nous verrons que la figure de l'ennemi (intérieur) fait partie des jeux politiques où les adversaires construisent des ennemis qui leur permettent de réaffirmer leurs propres engagements et de mobiliser des alliés. Dans le processus de construction d'un ennemi, l'attention se focalise moins sur les qualités personnelles et les tactiques employées par les uns et les autres que sur les traits caractéristiques intrinsèques de l'ennemi désigné (le juif, le libéral, l'immigré, etc.). Ainsi, l'étiquetage des ennemis favorise l'oubli des diversités et des distinctions individuelles en fixant l'attention sur tel ou tel rôle ou caractéristique symbolisant une menace : la couleur de la peau, la nationalité, l'ethnicité, les 
croyances, les modes de vie, etc. L'ennemi est alors manifestement inactif, sa passivité devient l'indice de ses menées souterraines pour saper les fondements de la sociétée ${ }^{17}$.

\section{L'immigré et l'ennemi intérieur : quel(s) lien(s) discursif(s)?}

Aux Etats-Unis le lien discursif entre ennemi intérieur et immigration est historiquement établi. En effet, l'irruption de l'idée de l'ennemi intérieur dans les discours politiques et sécuritaires américains est aussi vieille que la construction des Etats-Unis par les migrants fuyant l'Europe. Dès la première réglementation portant sur la question de naturalisation en 1790 et l'adoption des Alien and Sedition Acts ${ }^{18}$, l'idée de l'ennemi intérieur a fait son chemin. Au moment de l'adoption de cette législation, l'image de l'ennemi était représentée par l'étranger (alien) venu en particulier de l'Angleterre et de l'Irlande, pays ennemis du moment. Ces étrangers étaient soupçonnés d'importation d'idées subversives et de violation d'allégeance envers la nouvelle république. En effet,se focalisant sur un éventuel lien des migrants avec ces pays, les autorités craignaient un sabotage économique et idéologique dirigé par eux. Cette crainte avait même entraîné l'interdiction de la double nationalité. Comme l'explique Jeanne Chase, à cette époque, ce qui préoccupait les dirigeants américains c'était prioritairement l'intention des aliens.Que comptaient-ils faire sur le sol américain? Quelles étaient leurs vraies intentions? Comment pouvait-on les discerner facilement ? ${ }^{19}$.

On voit donc que dès ce moment, l'appellation ennemi de l'intérieur avait revêtu un sens politique et était associée aux notions d'allégeance, de loyauté, de trahison, de sabotage, de subversion et d'infiltration. Parmi ces notions, la question de l'allégeance a constitué une des préoccupations majeures des autorités américaines pendant la guerre avec la Grande-Bretagne. Elles ont soupçonné les citoyens naturalisés de continuer à affirmer leur allégeance au royaume ennemi. En se focalisant sur l'exemple de l'Américain John Watts qui avait choisi de devenir royaliste, les autorités ont présenté la trahison comme le pire des crimes contre l'Etat. Mais si dans leurs énoncés, la notion de trahison était pourvue d'une définition étroite, la notion de subversion restait dans le flou. Cette ambiguïté permettait aux autorités de bénéficier d'une grande marge de liberté dans leurs pratiques d'énonciation et de désignation. Le subversif n'avait pas de visage ou plutôt pouvait prendre une multitude de visages. La tâche des autorités politiques consistait donc à le démasquer, à l'identifier et à le désigner. Pour cela elles ont mis sur pied toute une technique de renseignements sur la loyauté et les intentions non seulement des migrants (plus particulièrement des migrants anglais ) mais aussi des citoyens naturalisés. Cette tâche a incombé aux douaniers et aux agents du recensement pendant les recensements de 1812 et 1813 effectués dans un climat de guerre et dont l'objet était le repérage et la surveillance des migrants britanniques ${ }^{20}$.

Après 1880, même si l'apport économique des migrants était fortement apprécié, une période de méfiance a commencé avec l'arrivée massive d'étrangers d'origine modeste. Comme en témoignent les discours de Jefferson et de Washington, on craignait que les nouveaux arrivants n'introduisent des valeurs contraires aux valeurs américaines, qu'ils maintiennent leur langue, leurs coutumes et leurs styles de vie ${ }^{21}$. Cette époque marque ainsi l'introduction d'un des principaux arguments récurrents des discours 
politiques et sécuritaires, à savoir, la question de la protection des valeurs américaines. Qu'entendait-on par "valeurs américaines " ? Conformément à la Constitution, cellesci portaient sur l'accès à la prospérité individuelle, le bien-être général, la justice et fondait une hiérarchie particulière de valeurs sociales et d'institutions politiquesà respecter. La crainte des Américains était (et l'est toujours) de savoir si les nouveaux arrivants pouvaient s'adapter à ces valeurs, s'ils pouvaient abandonner leurs propres valeurs considérées comme contraires aux valeurs américaines. Cette inquiétude marque ainsi le début de la problématisation de la migration en référence à la question de l'assimilation. On peut dire qu'à partir de là, la notion d'assimilation devint un enjeu politique et culturel de premier ordre.

La conséquence directe de cette nouvelle orientation a été la fixation de normes socioculturelles et l'établissement de critères pour déterminer qui est " désirable » et qui est « indésirable ». Ainsi, dès la fin de la guerre de 1812, les autorités commencèrent às'interroger sur la compatibilité des valeurs des migrants avec les valeurs et les normes américaines et à développer l'idée d'un seuil au-delà duquel l'assimilation deviendrait impossible. Ces barrières générèrent un système d'exclusion sur critères culturels et sociaux. Elles donnèrent lieu à l'établissement des listes de personnes indésirables et justifièrent le recours à des lois d'exception. Ainsi la première loi restrictive a été votée en 1872 interdisant l'entrée des prostituées et des condamnés. Plus tard, avec une loi adoptée en 1907 le Congrès a ajouté d'autres catégories à la liste des indésirables, comme les malades mentaux, les personnes atteintes de tuberculose, les enfants seuls (appelés enfants errants), les criminels. En 1917, une loi a porté sur l'interdiction des personnes reconnues coupables de «crime capital» comme la polygamie, la prostitution et a créé des zones d'exclusion. Et en 1918, suite à la révolution russe, la liste des indésirables a pris une tonalité politique en portant sur les personnes susceptibles d'importer les idées dites subversives (Anarchism Act). Plus tard, en 1921 et 1924, pendant la période de restriction, le Congrès a énuméré d'autres critères qui empêchaient les pauvres, les prostituées, les analphabètes, les malades, les anarchistes d'entrer sur le sol américain. C'est également en 1921 que le Congrès a fixé pour la première fois des quotas d'immigration par nationalité. Ceux-ci concernaient toutes les nationalités à l'exception des Canadiens, des Mexicains, des Haïtiens et des Dominicains. Il faut souligner que les quotas qui avaient été instaurés pour réduire l'immigration ont eu l'effet inverse, c'est-à-dire, le développement d'une immigration clandestine.

Parmi les politiques restrictionnistes, celle qui a le plus marqué le processus de construction de l'ennemi intérieur est sans doute la loi de 1952, appelée Mc CarrenWalter Act et préparée par les sénateurs démocrates Patrick Mc Carren et Francis Walter. Malgré son aspect libéral (elle supprimait la discrimination basée sur le sexe et la race), cette loi mettait en avant les inquiétudes sécuritaires liées à la menace communiste et instaurait un système de contrôle et d'exclusion afin de faire face à des activités dites "anti-américaines ». Elle établissait un lien entre les préoccupations de sécurité intérieure et le communisme en désignant comme ennemis les personnes déclarées ou supposées communistes. De même, en établissant un lien entre les activités anti-américaines et l'immigration, elle introduisait un système de préférences pour la sélection des migrants et mettait en place un dispositif d'exclusion et d'expulsion des indésirables (appelés les subversifs). Malgré le veto du président Truman et les critiques émises par les organisations humanitaires, la loi avait été adoptée à la Chambre avec une majorité de 278 voix contre 113. S'appuyant sur la thèse 
de la sécurité intérieure (au sens de internal security ${ }^{22}$ ), ses défenseurs avaient mis en avant l'argument de la préservation des Etats-Unis comme bastion contre le communisme. Ils avaient argué que la loi préservait l'homogénéité et permettait la défense des valeurs américaines. Cette focalisation sur les activités anti-américaines a donné lieu au développement d'une vaste production littéraire, artistique, cinématographique et théâtrale

Ainsi, on peut dire que dès les débuts de l'Union, l'introduction de l'idée de l'ennemi intérieur dans l'espace public s'est réalisée à travers l'établissement de deux interconnections discursives. Premièrement le lien alien/subversion (lien avec une puissance étrangère) mettant l'accent sur la question d'allégeance et les activités antiaméricaines. Deuxièmement, le lien immigrant/assimilation mettant l'accent sur la protection des valeurs américaines et la question de l'assimilation ${ }^{23}$.

Il est intéressant de noter que depuis cette date, ces deux interconnexions ont toujours structuré la problématisation de l'immigration et sa transformation en la figure de l'ennemi. Mais en fonction des contextes historiques et actuels, elles ont toutefois été accompagnées d'autres arguments comme la crainte de voir les migrants pauvres devenir une charge publique, la peur raciale et ethnique, l'invasion, le déséquilibre démographique, la criminalité, le trafic de drogue, etc.

Concernant ces derniers, celui qui a le plus marqué l'histoire de la "démonologie $»^{24}$ américaine est la référence à la race qui a opposé les blancs aux Amérindiens et aux peuples de couleur. Constituant la division essentielle de la vie américaine, la question raciale et ethnique a donné lieu à une longue liste d'exclus : Amérindiens, AfricansAméricans, Chinois, Japonais, Coréens, Philippins, Mexicains...

\section{La problématisation de l'immigration et l'exclusion en Californie}

Parmi les Etats de l'Union, la Californie est l'un de ceux où l'exclusion raciale est inscrite dans son histoire. Comme le suggère Annick Foucrier, l'histoire de la Californie s'est bâtie par la constante affirmation des blancs d'origine européenne de leur supériorité sur tout autre groupe racial ou ethnique ${ }^{25}$. Ainsi, à l'exclusion des Mexicains de leur terre natale suite au Traité de Guadalupe de 1848, s'ajouta l'interdiction faite aux Chinois d'accéder aux mines d'or, véritables ressources de l'économie californienne. Celle-ci s'est d'abord traduite dans les textes législatifs par l'article XIX de la constitution de 1879 et a abouti au Chinese Exclusion Act de 1882 qui constitue la première loi d'exclusion raciale. Elle fut complétée en 1884 par une loi qui a étendu la portée de l'exclusion à tous les Chinois et à leurs descendants quel que soit leur pays de résidence. Il ne faut pas oublier que pour justifier leur exclusion on accusa les Chinois d'être inassimilables en raison de leurs pratiques culinaires (" des mangeurs de riz et non de viande ») alors que tout avait été fait pour les marginaliser. Après les Chinois ce fut le tour des Japonais. Un mouvement anti-japonais commencé en 1888 a abouti à la loi de 1907 entérinant leur exclusion et à celle de 1915 (Alien Land Act) destinée à les empêcher d'acheter des terres en tant qu'étrangers non susceptibles de devenir citoyens. De 1942 à 1946, le Japon ayant été désigné comme leur ennemi principal, les autorités américaines ont interné de les ressortissants japonais vivant sur leur sol. Ensuite, c'est sur les Philippins venus les remplacer que se focalisèrent les peurs et les anxiétés du moment. 
A partir de 1910, date des troubles révolutionnaires dans leur pays, les Mexicains entrèrent dans la ligne de mire. Si pendant la Première Guerre Mondiale leur " côte " s'est un peu redressée en raison du manque de main d'œuvre (ils ont été appelés à travailler dans la construction des chemins de fer) et de l'éloignementdes migrants européens considérés comme des "fauteurs de trouble ", ils n'ont pas tardé à devenir eux aussi l'objet des pratiques d'exclusion. Ainsi, à partir des années 1920, victimes de préjugés raciaux et culturels, ils ont été cantonnés à des travaux subalternes et à des conditions de vie misérables.

En fait, l'attitude des Américains face aux Mexicains a toujours été pour le moins ambiguë. Ils ont été tantôt appelés à traverser la frontière, tantôt montrés du doigt et refoulés de l'autre côté du Rio Grande. Bienvenue pendant les périodes de nécessité (par exemple le programme Bracero de 1942), l'immigration mexicaine a été tributaire de crises économiques donnant lieu à des renvois massifs comme ce fut le cas en 1929, en 1941 (déportation de 112.000 personnes dont les deux-tiers étaient des citoyens américains) et en 1954 (Opération Wetback menée par un général de l'armée américaine).

Comme pour les autres migrants, la problématisation de la migration mexicaine et hispanique a été davantage accentuée à partir du moment où les Mexicains ont endossé la figure de l'étranger au sens défini par Simmel, c'est-à-dire, au sens d'« une personne arrivée aujourd'hui et qui restera demain $»^{26}$. Ainsi, quand les Mexicains ont commencé à faire venir leur famille et à s'installer définitivement, ils ont été considérés comme une menace pour "l'homogénéité culturelle et raciale Anglo-américaine ». Ils ont été empêchés de circuler en dehors de leur lieu de travail, interditsd'envoyer leurs enfants dans les écoles fréquentées par les enfants des WASP (White Anglo-Saxon Protestant), mis à l'écart de la société, bref, totalement marginalisés.

La transformation de l'immigration mexicaine en un problème politique et sécuritaire a été renforcée vers la fin des années soixante-dix par le discours sur la menace du Sud et la faiblesse des contrôles aux frontières face aux flux migratoires. Parmi les arguments rhétoriques, le plus récurrent est le thème de la perte de la souveraineté face aux flux migratoires et au trafic de drogue et le renforcement nécessaire des contrôles aux frontières pour y faire face. DeReagan à Clinton, ce thème est devenu un des arguments types du discours sécuritaire concernant les Mexicains.

Ainsi,les figures de l'ennemi intérieur en relation à l'immigration sont multiples. Cependant, il convient de noter que leur fabrication et le système d'exclusion qu'elles entraînent ne suivent pas une ligne historique continue et ne concernent pas tous les migrants de façon identique. Elles sont moins le résultat d'une histoire universelle et homogénéisante, que l'effet de processus singuliers de construction, d'objectivation et de subjectivation. Autrement dit, elles se réalisent dans des contextes socio-historiques particuliers. Ces processus se caractérisent par des discontinuités et des hétérogénéités. En effet, par exemple, si les Chinois ont été victimes de l'exclusion raciale au XIXe siècle, après avoir été désignés comme ennemis intérieurs pendant le Mc Carthysme par les Démocrates affaiblis, ils sont devenus des « amis » vers la fin du XXe siècle. De même, les Japonais, les ennemis du début et de la moitié du XXe siècle sont devenus aujourd'hui des partenaires incontournables. 


\section{La Proposition 187 et la construction de la figure de l'ennemi intérieur}

Lors des élections législatives du mi-mandat qui ont eu lieu le 8 novembre 1994, les Californiens ont non seulement renouvelé leurs représentants de la Chambre des Représentants et du Sénat ainsi que leur gouverneur, mais ont également voté, dans le cadre d'un référendum local, une proposition portant sur l'immigration clandestine appelée Proposition 187. Celle-ci était basée sur une initiative locale appelée Save Our States (S.O.S.) dont les auteurs les plus connus étaient Alan Nelson et Harold Ezel. Le premier avait été le directeur de l'INS ${ }^{27}$ sous Reagan, le second, le sous-directeur de la même agence. Pendant la préparation de l'initiative S.O.S., ils avaient travaillé pour l'organisation immigrée d'inspiration nativiste ${ }^{28}$, FAIR $^{29}$, qui a soutenu l'initiative et dont Nelson a été le directeur du bureau de Sacramento. Le groupe S.O.S. comportait également des anciens militaires, des anciens de la Border Patrol ${ }^{30}$, des hommes d'affaires, des individus appartenant à des associations locales.

La question qui avait été préparée par ce groupe et soumise au vote des Californiens était simple et directe : « Pour faire face aux problèmes économiques et à la criminalité montante faut-il priver les immigrés clandestins du bénéfice des services sociaux et éducatifs de la Californie? ». Les Californiens ont répondu "oui » avec une grande majorité de 59,1\% des voix.

Cette Proposition avait été préparée dans l'Etat de Californie pour faire réélire le gouverneur Pete Wilson (Rép) qui a su exploiter les préoccupations économiques et sécuritaires du pays touché par la perte de centaines de milliers d'emplois à la suite de la réduction des effectifs militaires après la fin de la Guerre Froide. Selon la Proposition 187, la responsabilité de cette situation incombait aux étrangers en situation irrégulière dont la majorité était composée de Mexicains qui traversaient, facilement et sans documents, la frontière du sud de la Californie. Pour le gouverneur Wilson, les clandestins ne se rendraient en Californie que pour bénéficier des services publics sociaux et éducatifs gratuits, ce qui coûtait à l'Etat plus d'un milliard de dollars par an. Pour y faire face, il fallait non seulement leur refuser l'accès à ces services mais obliger les enseignants, le personnel hospitalier et le personnel des services sociaux à vérifier les documents des demandeurs et dans le cas où ceux-ci seraient dépourvus de papiers, les dénoncer à l'INS. La loi allait même plus loin en stipulant l'expulsion des enfants clandestins vers leur pays d'origine.

Une des caractéristiques majeures du processus de désignation des immigrés clandestins comme cause de tous les maux et la principale menace contre l'Etat, était sa présentation en termes $d$ '«urgence sécuritaire» nécessitant le recours aux lois d'exception et aux mesures administratives et politiques expéditives. Ce que fit le gouverneur Wilson en déclarant "l'urgence de trouver une solution au problème de l'immigration ». Ce qui a entraîné l'adoption d'un ensemble de mesures allant de la mise hors la loi (« les clandestins commettent un acte illégal »), à l'expulsion en passant par la perte d'emploi et du logement, l'exclusion de la scolarité, la dénonciation et l'inscription dans des fichiers informatiques. En effet, concernant cette dernière mesure lors de leur expulsion, les immigrés clandestins étaient fichés dans les bureaux des services sociaux, à l'INS et à la Border Patrol, et leurs empreintes digitales étaient conservées par ces mêmes services. 
Très vite les discours qui ont été produits lors de l'adoption de cette loi ont débordé le seul cadre de l'immigration illégale pour porter sur l'immigration en général. C'est l'immigré tout court et en particulier celui qui venait des pays du Sud qui a été l'objet de ces discours. C'est lui qui a été présenté comme devant faire l'objet d'une préoccupation sécuritaire, c'est-à-dire comme la nouvelle figure de l'ennemi menaçant la société californienne.

Pour certains, le contexte de "crise de l'ennemi » apparu avec la fin de la bipolarité explique le ralliement d'un grand nombre d'acteurs comme les agences de sécurité et les politiciens de droite et de gauche à la fabrication de la nouvelle figure de l'ennemi. Ce qui a donné lieu à des débats profonds sur la définition de l'identité américaine et à la remise en cause des définitions traditionnellement acquises. Pour la gauche, depuis les années 1960, l'identité nationale était synonyme d'identité populaire commune basée sur les droits civiques et la démocratie. Pour la droite, opposée au mouvement des droits civiques, l'identité devait au contraire reposer sur les valeurs culturelles et religieuses et protéger la civilisation américaine de l'invasion des barbares.

Mais avec la fin de la menace communiste, ce qui pouvait souder et caractériser les Américains ne pouvait être une nouvelle figure ayant trait à la question des valeurs. Cette orientation, même si elle était plus défendue par les Républicains et les ultras de Pat Buchanan que par les Démocrates, ne laissait pas ces derniers insensibles. Dès les années 1980 de nombreux discours, venant d'acteurs qui n'étaient pas nécessairement des alliés, avaient commencé à désigner les immigrés comme cause de problèmes divers. On peut citer les discours sur la sécurisation de la frontière américanomexicaine, les discours sur la criminalité et le trafic de drogue, dont les énoncés construisaient un lien avec l'immigration. De même, on peut citer les prévisions démographiques alarmistes, selon lesquelles en l'an 2005, les Californiens blancs deviendraient une minorité au détriment des Hispaniques. Dans ce processus discursif, il y a également des influences nativistes et les nouvelles lectures de l'ethnicité en termes de division sociale et culturelle qui viennent souvent en lieu et place d'une analyse de classe. Enfin il faudra citer le débat sur le Welfare et le coût de l'immigration qui est parti de San Diego State University et qui s'est poursuivi par la publication en 1993 du rapport controversé, mais néanmoins toujours cité de Donald Huddle qui affirmait que les immigrés (légaux et clandestins) coûtaient à l'Etat plus qu'ils ne lui rapportaient ${ }^{31}$.

Pendant que ces discours sur la menace étaient énoncés, se produisait un autre discours, plus moraliste, reprenant les idées des valeurs familiales, traditionnelles et religieuses. Le contrat sur l'Amériqueque proposait Newt Gingritch mettait en avant l'idée de la responsabilité et des valeurs. Inspiré des thèses communautaristes de droite, ce discours, critiquant la politique égalitariste du libéralisme politique, proposait de distinguer entre les droits et les devoirs et de privilégier ces derniers au détriment des premiers. Dorénavant, la citoyenneté devait s'articuler sur l'idée de la responsabilité. Il fallait travailler, protéger les valeurs familiales et religieuses et être responsable plutôt que de réclamer des droits. Ceux qui ne respectaient pas ce contrat, se marginalisaient dans des ghettos où ils ne vivaient que de l'aide sociale.

Inscrite dans un contexte particulier, la Proposition 187 est également le résultat des jeux politiques et bureaucratiques. 


\section{Les jeux politiques et bureaucratiques}

Les jeux politiques et bureaucratiques ont fait émerger comme légitime cette interconnexion entre différents arguments et ont permis la polarisation sur une nouvelle figure de l'ennemi.

Quand on analyse les auteurs qui ont préparé la Proposition 187 ainsi que les discours sur la menace qui ont été produits, on trouve à côté du gouverneur Wilson, le Parti Républicain qui l'a soutenu, les organisations anti-immigrés qui ont fait du lobbying en sa faveur et d'autres acteurs comme les agences de sécurité telles que la Border Patrol, les militaires et les associations locales. On trouve également des politiciens qui n'appartiennent pas seulement à la droite mais aussi à la gauche comme Diane Feinstein, sénateur Démocrate de Californie et ancien maire de San Francisco. Cette dernière, oubliant ses discours des années 1980 où elle présentait San Francisco comme "le sanctuaire des réfugiés venus du Salvador et de l'Irlande », n'a pas hésité à établir un lien entre immigration, criminalité et trafic de drogue et focalisa son discours sur le besoin de renforcer les contrôles à la frontière pour faire face au danger de l'immigration clandestine. Bien que Diane Feinstein n'eut pas voté «oui » à la Proposition 187, dans les discours qu'elle a tenus, elle a reproduit pratiquement les mêmes arguments que ses adversaires politiques.

Il faudra aussi citer le revirement au centre du discours politique du président Clinton qui, après le succès des Républicains au Congrès a déclaré « l'immigration clandestine comme un problème majeur » et a insisté sur la nécessité de renforcer les contrôles à la frontière américano-mexicaine. A peu près tous les politiciens du parti Démocrate ont suivi cette même voie, légitimant la problématisation de l'immigration sans toutefois s'interroger sur les présupposés sur lesquels elle se basait et les arguments rhétoriques qu'elle déployait. Ainsi aux yeux de toute la classe politique, l'immigration en tant que telle était devenue un problème. Tous étaient en compétition pour produire un discours correspondant aux peurs du moment et pour proposer des remèdes. Remarquons que cette (re)production discursive n'était pas le résultat d'une désignation collective et consensuelle de l'immigration comme adversaire commun par tous les acteurs. Elle constituait plutôt le point focal sur lequel convergeaient les insécurisations économiques, démographiques, policières, militaires, identitaires voire même écologiques.

Ainsi, la similitude des discours et des problématisations de l'immigration ne doit pas être interprétée comme résultant d'un complot, mais comme des jeux et des tactiques politiques entre acteurs, générés par des enjeux de positionnement ainsi que de protection des intérêts dans le contexte de l'après-Guerre Froide. Pendant qu'ils criminalisaient les immigrés, ces acteurs proposaient une variété de solutions. Ce qui a provoqué à la fois un jeu de coopération et de rivalité. Comme exemple, nous pouvons citer les différences de vue pendant la campagne en faveur de la Proposition 187 entre l'INS et les autres acteurs qui tout en présentant des solutions allant dans le sens du renforcement de la frontière et du déploiement de plus d'agents sur place, se distinguaient dans leur façon de désigner l'ennemi. Ainsi, en faisant attention de distinguer entre l'immigré légal et l'immigré clandestin, l'INS s'est focalisé sur ce dernier et a tenu un discours intégrationniste pour les premiers en les incitant à se faire naturaliser. Ce qui allait à l'encontre des discours tenus par la FAIR qui refusait catégoriquement la naturalisation des non-Européens. L'INS a également remis en 
cause les chiffres publiés par le gouverneur Wilson et FAIR sur l'immigration clandestine.

De son côté, la Border Patrol cherchait à attirer l'attention sur l'urgence d'un renforcement des contrôles de la frontière en citant des chiffres sur le nombre de passages clandestins, des informations sur les réseaux de passeurs. Par là son objectif était de montrer la crédibilité de ses thèses afin de demander l'augmentation de son budget et de ses effectifs. On peut également évoquer les relations entre les militaires et la Border Patrol déployés pour la surveillance et le contrôle de la frontière. Leur lecture de la menace venant du Sud avait permis de créer une coopération a priori contre nature entre eux donnant lieu à la constitution de nouvelles alliances et réseaux. Comme ils travaillaient ensemble au contrôle et à la surveillance de la zone frontalière, ils avaient plutôt intérêt à apparaître en position d'autorité pour obtenir les avantages qu'ils réclamaient du gouvernement.

Il faut également rappeler le rôle des associations locales (grassroots organisations) dans ces jeux de positionnement ainsi que dans la sécurisation de l'immigration.

Le mouvement grassroots qui est très actif surtout au sud de la Californie sensibilise et mobilise l'opinion et influence la mise sur agenda politique des questions d'immigration. Avec des membres corrélés aux milieux politiques et sécuritaires, il permet la constitution d'un lien entre ces derniers et les citoyens. Comme on peut le voir avec l'exemple de la FAIR, il créé un réseau de relations très actif et influence les luttes de positionnement entre acteurs. Ainsi la FAIR, par les discours qu'elle a produits et par ses réseaux au niveau local et fédéral, est intervenue indirectement dans les luttes entre la Border Patrol et l'INS. Les premiers réclamant plus d'autonomie ne voulaient plus rester sous la tutelle des seconds. Mais ces derniers, voulant conserver leur position d'autorité ont souvent rappelé à l'ordre la Border Patrol, surtout lorsque celle-ci voulait mener à la frontière une politique plus indépendante et plus en phase avec les militaires. Ainsi, tout en défendant l'augmentation du nombre des agences de sécurité déployées à la frontière, la FAIR a exprimé une attitude ambiguë vis-à-vis de l'INS. Elle n'a pas raté une occasion pour le critiquer d'inertie, allant même jusqu'à demander son démantèlement en proposant la création d'une nouvelle agence sous la tutelle des ministères de la justice, du travail et du département d'Etat.

\section{Les procédés communicationnels}

Pour faire passer le message de la Proposition 187, d'importants moyens de communication avaient été mis en œuvre : des collectes de fonds et de signatures, des campagnes de publicité et surtout des spots télévisés. Ainsi les Californiens ont vu en prime-time des images montrant des Mexicains en train de franchir la frontière à l'aube. Une voix off accompagnait ces images en disant tout simplement « ils arrivent ». Il y avait aussi des images montrant des femmes d'origine hispanique descendant de l'avion et se précipitant dans les hôpitaux pour accoucher d'enfants qui allaient devenir automatiquement américains grâce à la loi qui attribue la nationalité américaine à chaque enfant né sur le sol américain.

Tout avait été ainsi mis en œuvre pour sensibiliser les électeurs qui avaient été ciblés par classe d'âge et de revenu. L'une des principales cibles était les retraités riches qui sont importants en nombre (la Californie reçoit la majorité des retraités aisés des EtatsUnis) et en influence (ils constituent des réseaux influents dont le plus connu est 
l'American Retiree Association). Auprès de ces derniers, les références argumentaires relatives aux impôts locaux et au financement de l'école publique ont eu un impact considérable. Car, les retraités n'ont plus d'enfants en bas âge et s'ils ont des petits enfants, ceux-ci ne résident pas nécessairement dans le même Etat qu'eux. Aussi, comme l'école publique est financée par les impôts locaux et comme les retraités sont ceux qui payent le plus d'impôts en raison de la valeur élevée de leurs habitations, ils ont été plus sensibles à la Proposition 187.

On peut dire que l'une des raisons de l'échec de la campagne des opposants à la Proposition 187 a été son incapacité à cibler un électorat particulier. Leur campagne était destinée à un public universel. Elle a déployé des références et des thèmes généraux comme les droits de l'homme ou la lutte contre la discrimination. Et si certaines organisations ont voulu faire une campagne ciblée, leur ciblage a été ethnique en se focalisant uniquement sur la communauté mexicaine. Ce qui a eu pour conséquence de présenter la Proposition 187 comme une loi qui ne concernait que cette communauté et non tous les habitants de la Californie.

\section{Les procédés argumentatifs}

Pour construire l'immigration comme un problème, les discours développés ont établi une corrélation entre l'immigration, l'Etat-providence (welfare), la fraude, la criminalité, la protection des frontières, le terrorisme, le trafic de drogue, les incivilités et la violence urbaine donnant lieu à un continuum sécuritaire. Et c'est d'abord le clandestin, puis l'immigré en tant que tel, qui ont été désignés comme la figure incarnant l'ennemi. Il nous faudra noter ici avec Edelman que l'amalgame de problèmes divers sous les traits d'un ennemi censé les représenter est un phénomène politique banal et une manière puissante pour rallier les partisans à une cause. Ces amalgames peuvent servir à édifier toutes sortes de coalitions politiques. Car «plus le visage de l'ennemi prête à équivoque, plus la compréhension des situations pernicieuses est malaisée et plus les alliances politiques dirigées contre cet ennemi sont étroitement soudées $\rrbracket^{32}$.

On peut dire que cette construction discursive était motivée par deux préoccupations majeures : premièrement, convaincre du danger de l'immigration porté par les clandestins prêts à tout et constituant par leur présence sur le sol américain une charge publique pour la communauté. Deuxièmement, légitimer la menace faite par les migrants aux valeurs culturelles américaines en fournissant comme exemple leur inassimilation et leurs revendications multiculturalistes. Celles-ci ont été exprimées à travers une procédure rhétorique en appelant aux rationalisations et aux arguments suivants : la perte de la souveraineté et la nécessité de renforcer les contrôles aux frontières, la corrélation entre l'immigration clandestine, la criminalité et le trafic de drogue, le lien entre l'immigration, le chômage et la crise de l'Etat-providence, la crainte de l'invasion, l'absence d'assimilation des migrants, les revendications multiculturalistes et l'atteinte aux valeurs américaines ${ }^{33}$.

Parmi ces arguments, ceux qui ont eu le plus d'impact sur les électeurs sont le lien établi entre les droits sociaux et la crise de l'Etat-providence et l'inassimilabilité des migrants et la peur du multiculturalisme. 


\section{Le lien entre les droits sociaux, la crise du welfare et les impôts locaux}

Cette configuration énonciative part du présupposé que les droits sociaux constituent un attrait pour les migrants originaires des pays pauvres. Même si cet argument ne repose sur aucune analyse sérieuse, dans le raisonnement des auteurs de la Proposition 187, il était devenu la norme. Dans ce raisonnement, l'immigration massive était l'œuvre de pauvres gens qui ne venaient aux Etats-Unis que pour bénéficier des droits sociaux « généreusement attribués ». De plus, outre leur caractère de facteur d'appel, les droits sociaux permettraient à ceux qui étaient clandestinement «infiltrés " d'y rester et de survivre au détriment du contribuable américain qui devrait supporter le coût des dépenses sociales en payant de sa poche. De même, la scolarité gratuitement fournie était un moyen de faire rester les clandestins sur le sol californien puisqu'ils penseraient que leurs enfants ne pourraient pas être expulsés. Ce qui, en bout de chaîne, causerait le surpeuplement des classes et la baisse du niveau de l'enseignement.

Expliquer les problèmes économiques en désignant les immigrés comme responsables est une tendance bien connue en temps de crise. Mais depuis le début des années 1990, les conséquences économiques de l'immigration ont été analysées à travers un changement thématique et sémantique. On est passé d'une analyse en termes de marché de l'emploi à une analyse de l'Etat-providence et des impacts fiscaux de l'immigration sur celui-ci. Avec un réseau sémantique désignant les migrants comme une " charge publique ", une " charge supplémentaire pour les budgets familiaux ", un "poids fiscal inutile", les discours se sont focalisés sur l'impact fiscal négatif de l'immigration sur les citoyens nationaux.

Cet argument constitue une nouveauté par rapport aux discours produits au XIXe siècle où l'on évoquait également le thème de "charge publique " pour les pauvres et les prostituées. Mais avec la Proposition 187, ce qui est nouveau, c'est la perception que les migrants constituent une charge pour le contribuable et qu'en conséquence ils doivent être exclus du bénéfice des droits sociaux ainsi que de la scolarité gratuite. Il faut dire que l'argument ne s'arrête pas là, mais va jusqu'à mobiliser le citoyen national pour qu'il refuse de payer des impôts tant que l'Etat ne résout pas le problème de l'immigration. Ce type d'argumentation liant droits sociaux, fiscalité et responsabilité de l'Etat n'avait pas été déployé auparavant dans les discours nativistes. Entré dans les discours avec la Proposition 187, il s'est accompagné sur le plan sémantique du glissement du citoyen vers le contribuable, faisant de lui la référence principale de la vie civique. Dorénavant c'est lui qui fixe les règles du jeu et menace de rompre le contrat qui le lie au gouvernement tant que celui-ci ne satisfait pas ses désirs.

On peut dire que cet argument et les glissements sémantiques qui l'ont accompagné ne se sont pas arrêtés à la Proposition 187. Ils ont été redéployés et élargis avec l'adoption d'une loi sur le Welfare qui s'est largement inspirée de la Proposition 187. La loi intitulée Personal Responsablity and Work Opportunity Reconciliation Act(PRWOA) a ciblé non seulement les clandestins mais aussi les migrants légaux qui vivent des droits sociaux. Par exemple, les migrants pauvres et les migrants âgés qui perçoivent le SSI (Social Security Income) ont été désignés comme constituant une "charge publique " pour la communauté et comme étant des "personnes non méritantes vivant de la générosité des citoyens qui travaillent et payent des impôts ». Notons que ces énoncés ont été fabriqués dans le contexte des idées développées par le Contrat avec l'Amérique 
que nous avons évoqué précédemment, qui fixait de nouvelles valeurs morales et de nouvelles normes sociales. Ainsi, la tendance a été de relier l'aide sociale aux plus démunis à une surveillance morale stricte et punitive par exemple en contrôlant la vie familiale, en punissant l'avortement, en prévoyant l'internement d'office des enfants des mères célibataires, en responsabilisant les pères, etc.

\section{L'inassimilabilité des migrants et la peur du multiculturalisme}

Cet argument a surtout visé les Mexicains et il a été particulièrement développé par les organisations anti-immigrés, comme la FAIR, qui ont mené leur action au niveau local. Celles-ci ont construit leur argumentaire à partir du postulat de la différence culturelle qui persiste malgré la vie en commun dans la société. Comparant l'immigration mexicaine aux vagues de migration précédentes, en particulier à celles des Européens, elles ont accusé les Mexicains de ne pas rompre avec leurs traditions culturelles et de refuser de s'assimiler à la société américaine. Comme exemples, elles ont cité l'utilisation de l'espagnol, la fréquence des voyages vers le pays d'origine, le faible taux de mariages mixtes, le travail dans les emplois ethniques, la ghettoïsation, etc. Ce qui à leurs yeux donnerait lieu à la constitution d'une communauté isolée sans véritable interaction avec le reste de la société et mènerait à terme à la "balkanisation $»^{34}$ des Etats-Unis. Comme nous l'avons déjà mentionné, le thème de l'inassimilabilité des Mexicains n'est pas nouveau. Les Mexicains ont toujours été stigmatisés sous des traits culturels élaborés dans une approche essentialiste et fixiste en faisant référence aux traditions, à la religion aux modes de vie qui seraient contraires à ceux des Américains. Comme le montre le discours prononcé par un officiel en 1911 devant la Commission Dillingham sur l'immigration, les Mexicains ont été perçus comme «constituant une bonne main d'œuvre, mais pas facilement assimilables » et étant par conséquent " moins acceptables comme citoyens que comme ouvriers temporaires $»^{35}$.

Depuis la moitié des années 1980, le multiculturalisme qui au départ était un débat philosophique, est entré dans les discours sécuritaires comme constituant une menace à la culture et à l'identité américaines. Réduit au multilinguisme, à la réécriture des livres d'histoire, au refus des cours de civilisation européenne dans les universités, à l'Affirmative Action, il a été accusé de rejeter les racines européennes de l'identité américaine et d'introduire des références non-européennes au nom de la diversité culturelle. Appréhendé dans un sens différentialiste comme l'affirmation de différences culturelles menant aux revendications de reconnaissance politique, il a été accusé d'inciter à la constitution de communautés exclusivistes renfermées sur elles-mêmes et porteuses de cultures incompatibles avec la culture américaine. C'est ainsi que Samuel Huntington, le père du paradigme du clash des civilisations, l'a accusé d'encourager l'infiltration de la civilisation américaine par des valeurs étrangères et de mener les Etats-Unis à la «balkanisation». D'autres comme Michael Lind, défenseur d'un nationalisme de valeurs communes, ont parlé de la «brésilianisation» du pays ${ }^{36}$.

Comme le remarquent Yosef Lapid et Friedrich Kratochwill ${ }^{37}$, nous assistons avec ces discours au retour des arguments culturels dans l'interprétation de l'identité nationale. Ceci donne lieu à la perception des migrants comme «l'Autre culturel» qui, par sa présence, dérange une société culturellement unie, harmonieuse et homogène. En conséquence, il transforme les interactions entre les migrants et les nationaux en un 
conflit culturel et donne lieu à une représentation de la société en termes antagonistes, en opposant le "Nous" et les "Autres". Selon David Campbell, les thèses de balkanisation ou de désunion ainsi que les discours sur la menace permettent d'établir une relation entre ce qui est perçu comme l'élément constitutif et résistant d'une identité sécurisée à l'intérieur de la nation avec les menaces identifiées et localisées à l'extérieur de l'Etat. Ce lien permet de se focaliser sur l'idée de l'étrangeté en tant que situation où l'on se trouve à la fois à l'intérieur et à l'extérieur ${ }^{38}$. En d'autres termes, le danger est considéré comme étant localisé simultanément à l'extérieur et à l'intérieur. Ce qui permet de constituer une relation discursive entre le multiculturalisme et l'immigration et de la présenter comme une menace portant atteinte à la sécurité identitaire des nationaux.

Ainsi, pendant la campagne en faveur de la Proposition 187, un grand nombre de discours s'est focalisé sur ce lien en citant l'exemple des Mexicains qui en conservant leurs caractéristiques culturelles et en revendiquant plus de reconnaissance auraient des visées séparatistes et constitueraient un danger pour la sécurité nationale et identitaire des Californiens.

Les discours sur le danger multiculturaliste ont permis le retour de la référence à l'allégeance et à la loyauté et ont réveillé la vieille crainte de la double nationalité. Profitant de ce contexte, le gouverneur Wilson, soutenu par la FAIR a même proposé de réformer la loi sur la nationalité demandant la suppression de la double nationalité. Il a également suggéré - comme les Français l'ont fait en 1993 avec les lois Pasqua d'empêcher l'acquisition automatique de la nationalité américaine par les enfants d'étrangers nés sur le sol américain. De même, les nativistes, à l'instar de Peter Brimelow ${ }^{39}$ ont proposé d'instaurer des cartes d'identité nationale à la française, allant à l'encontre de la tradition américaine selon laquelle l'identité est une affaire privée qui ne saurait être soumise à l'inspection de la puissance publique.

\section{Les discours de résistance}

Les discours en faveur de la Proposition 187 ont provoqué de nombreuses réactions et donné lieu à la production de discours de résistance de la part de différents acteurs tels que les organisations de défense des droits des Mexicains-Américains, ou des ChinoisAméricains, les juristes experts en immigration, les ONG qui défendent les migrants, les églises, les milieux d'affaires et même certains think tanks conservateurs. On peut dire que ces acteurs dont la nature, la mission et les discours sont différents en temps normal, ont émis des critiques pratiquement convergentes sur la Proposition 187 et ses conséquences sur l'immigration. Ainsi a-t-on vu les milieux d'affaires s'exprimant dans les colonnes du quotidien Wall Street Journal ou le Cato Institute, le think tank conservateur, s'opposer à la loi et défendre une politique migratoire libérale.

Les critiques portées à la loi étaient structurées autour de trois arguments majeurs. Le premier argument, le plus cité, était juridique. Défendu par les juristes comme la Lawyers Committe for Civil Rights, et les organisations de défense des immigrés comme la Coalition for Immigration Rights and Refugee Services, celui-ci rappelant la tradition des droits civiques reprochait à la loi le système d'exclusion qu'elle instaurait et mettait en avant son caractère anticonstitutionnel.

Le deuxième argument était le caractère raciste et anti-mexicain de la loi. Cet argument était défendu par les organisations de défense des intérêts de la communauté 
mexicaine et mexicaine-américaine comme le MALDEF ${ }^{40}$ qui joue également un rôle de groupe de pression et de formation des leaders communautaires, ou le COPS $^{41}$ qui permet de faire entendre les revendications de cette communauté auprès des élus. Selon ces organisations, la Proposition 187 était particulièrement dirigée contre la communauté mexicaine et était accompagnée de préjugés racistes forgeant des images négatives et reproduisant des stéréotypes. Pour faire entendre leurs critiques ils ont organisé des manifestations comme celle qui a eu lieu à Sacramento au début de l'année 1994 ou des marches comme celle qui s'est déroulée à Los Angeles avant le vote de la loi. Mais cette dernière action a été détournée de son objectif et a été transformée en une marche anti-américaine avec des Mexicains brandissant le drapeau de leur pays. Ce qui a provoqué une réaction nationaliste de la part d'électeurs américains blancs.

Le troisième argument était que la loi portait atteinte à la tradition libérale d'immigration du pays. Défendu par les milieux d'affaires et les églises cet argument présentait l'immigration comme un droit naturel pour l'être humain (thèse des églises) et comme un bien pour l'économie du pays. Les milieux d'affaires qui, d'habitude expriment leur sympathie envers les Républicains, n'ont pas hésité à montrer leur désaccord sur cette question arguant les avantages économiques de la poursuite de l'immigration. Peut-on toutefois dire que tous les milieux d'affaires ont soutenu cette thèse ? Il est intéressant d'étudier à cet égard l'attitude de la Silicon Valley qui emploie un grand nombre de travailleurs immigrés. Il y eut certes des compagnies qui ont soutenu une politique restrictive, voire même nativiste. Il y eut même des compagnies qui ont participé au financement de la Proposition 187, mais en général, la Silicon Valley est restée sur une position libérale. Il convient de remarquer que quelques années après l'adoption de la Proposition 187 et d'une nouvelle loi fédérale plutôt restrictive sur l'immigration en 1996, c'est à l'initiative des hommes d'affaires de la Silicon Valley que le gouvernement a adopté une loi autorisant l'augmentation du nombre de visas attribués à des étrangers hautement qualifiés travaillant dans cette région (high tech visas). Comme on peut le constater, leur politique libérale concernait plutôt les travailleurs qualifiés, mais pendant la campagne de la Proposition 187, ils n'ont pas émis de critiques contre les véritables cibles de la loi, c'est à dire les Mexicains à revenu faible ou moyen.

Aucun de ces arguments n'a porté sur l'impact de la loi sur la société californienne en général et sur le type de société dont les contours se dessinaient à travers elle. Ils ont défendu des thèses soit universalistes (droits de l'homme, droits civiques, droit de l'immigration), soit communautaristes (la défense de la communauté hispanique), mais ne sont pas parvenu à structurer un discours portant sur les véritables enjeux de la Proposition 187 et de ses impacts sur la Californie. Car au fond, en construisant des ennemis, en forgeant des trames narratives singulières, en instaurant un système d'exclusion, on ne faisait que se définir soi-même. Mais ceci n'a pas donné lieu à une réflexion profonde sur cette question.

De même, ces discours ne sont pas parvenus à organiser une véritable opposition à la loi. De nombreux facteurs peuvent expliquer cet échec : la diversité des acteurs et le manque de communication entre eux, leur mobilisation tardive, le peu de fonds mis à leur disposition malgré le soutien des milieux d'affaires, la division au sein des organisations de défense des immigrés et l'absence d'action au niveau local comme l'ont fait les défenseurs de la loi. Il faut bien remarquer que les discours de résistance n'ont pas provoqué une grande participation des communautés hispaniques aux 
élections de 1994, car un grand nombre de résidants ne possédait pas la nationalité américaine. Parmi ceux qui l'avaient, seulement $8 \%$ ont participé au vote.

La loi a également engendré des divisions au sein des immigrés et des communautés. Certains d'entre eux ont épousé les thèses des Républicains et criminalisé les clandestins et ceux qui vivent des bénéfices du Welfare. Ainsi, 57\% d'Asiatiques, 56\% d'Africains-Américains et $37 \%$ d'Hispaniques ont voté en faveur de la loi.

Les retournements de situation ne se sont produits que quelques années plus tard. Sur le plan civique, la loi a eu un impact mobilisateur auprès des résidants hispaniques qui ne s'étaient pas fait naturaliser et qui ne s'étaient pas impliqués dans la politique locale. Comme on a pu le constater avec les élections de 1998, il y a eu une forte augmentation du nombre d'Hispaniques naturalisés ainsi que de leur inscription sur les listes électorales. Les élections se sont soldées par la nomination du Démocrate Gray Davis au poste de gouverneur, et pour la première fois, d'un latino-américain, Cruz Bustamante (D) au poste de vice-gouverneur.

Après un moment d'hésitation, le gouverneur Davis a décidé de ne pas appliquer la Proposition 187. Ce qui a provoqué une forte réaction de la part des défenseurs de la loi qui au niveau local n'ont plus d'arguments juridiques suffisants pour la faire appliquer. Paradoxalement c'est au niveau fédéral que les orientations fixées par elle, et les dispositifs proposés ont été repris.

Après le passage de la Proposition 187, le juge fédéral a annulé son application pour cause d'anticonstitutionnalité (la Constitution prévoit la scolarisation de tous les enfants nés sur le sol américain). Mais, malgré cette annulation, la loi n'a pas disparu pour autant du champ politique ainsi que des espaces discursifs et a inspiré des lois fédérales sur l'immigration et le Welfare. Ainsi, ses dispositions portant sur l'immigration clandestine, le contrôle de la légalité du séjour des bénéficiaires des droits sociaux, le refus de l'aide médicale aux clandestins sauf pour les urgences ainsi que la suppression de leur bénéfice des droits sociaux ont servi de modèle aux lois fédérales adoptées en 1996 : la loi sur l'immigration (Illegal Immigration Reform and Immigrant Responsability Act) et la loi sur le Welfare (Personal Responsability and Work Opportunity Act) que nous avons évoqué précédemment. Par ailleurs, une troisième loi portant sur le terrorisme (Antiterrorism and Effective Death Penalty Act) adoptée la même année, s'est inspirée de ces dispositions portant sur la criminalité, ainsi que des discours qui l'ont accompagné et qui ont établi un lien entre l'immigration, la criminalité, le terrorisme et le trafic de drogue. Au fond, on peut dire que ces trois lois ont reproduit le « continuum sécuritaire $»^{42}$ amalgamant des questions diverses dans les traits d'un ennemi intérieur censé les représenter. Cet ennemi c'est l'immigré qui est aussi le pauvre, le marginal, le profiteur des droits sociaux, le fraudeur, le criminel et celui qui porte atteinte à l'harmonie de la communauté. Il est présenté comme constituant une menace aux valeurs culturelles, à l'identité, à l'ordre public, à la sécurité et à l'ordre moral. 


\section{NOTES}

1. Murray Edelman, Pièces et règles du jeu politique, Paris, Editions du Seuil, 1991.

2. Ibid., pp.21-22.

3. Elle va à l'encontre d'une décision de la Cour Suprême rendue en 1982 (Pyler vDoe) qui garantit le droit d'accès à l'éducation publique gratuite pour tous les enfants, même clandestins, se trouvant sur le sol américain.

4. Nous empruntons cette expression à Patrick Champagne, Faire l'opinion, le nouveau jeu politique, Paris, Editions de Minuit, 1990, p.8.

5. Gilles Deleuze, «Qu'est-ce-qu'un dispositif?, in Michel Foucault philosophe, Rencontre Internationale, Paris, 9,10,11 janvier 1989, Paris, Le Seuil, 1989.

6. Pour plus de développements, voir le texte de Yves Viltard dans ce numéro.

7. Michael Hardt, "La société mondiale de contrôle », in Eric Alliez (Ed), Gilles Deleuze une vie philosophique, Rencontres internationales Rio de Janeiro- Sao Paulo, 10-14 juin 1996, collection Les empêcheurs de penser rond, 1998, pp.363-364.

8. Ibid., p.364.

9. David Campbell, Writing Security, United States Foreign Policy and the Politics of Identity, Minneapolis, University of Minnesota Press, 1992.

10. Sur ce sujet voir essentiellement Barry Buzan, Peoples, States and Fear, Brighton, Harvester, 1993 et Ole Waever, Concepts of Security, Copenhagen, Institute of Political Science, University of Copenhagen, 1997.

11. Aux Etats-Unis, l'expression sécurité intérieure (internal security) n'est pas utilisée dans le sens où elle est employée en France. En effet, « internal security » renvoie à la commission sur la « sécurité intérieure » créée sous le maccarthysme pour réprimer les comportements politiques anti-américains. Dans ce texte, nous employons l'expression "sécurité à l'intérieur» pour désigner la notion de sécurité intérieure. Aux Etats-Unis, cette notion dont les contours ne sont pas bien précis ne porte pas seulement sur le contrôle de la délinquance et de la criminalité, mais aussi renvoie à la surveillance des menaces possibles à la culture, à l'identité, à l'environnement etc. Voir Amos Jordan, William Jesse Taylor, American National Security Policy \& Processes, Baltimore, John Hopkins University Press, 1989.

12. Alexandre Koyré, La cinquième colonne, paru initialement en 1945 à New York, dans le numéro II-III de Renaissance, revue publiée par l'Ecole Libre des Hautes Etudes de New York, Nouvelle édition française, Paris, Editions Allia, 1997, p.14.

13. Koyré écrit : «C'est au général Franco - dont ce sera, sans doute, la création la plus populaire et la plus durable - que nous devons (le terme ' cinquième colonne'). C'est lors de la guerre civile espagnole, ou mieux, lors de la contre-révolution espagnole, lors de la marche des armées franquistes sur la capitale, que 'la cinquième colonne' vit le jour. Les quatre colonnes qui s'approchent de Madrid seront, a dit le général Franco, aidées par une cinquième qui s'y trouve déjà. L'image frappa la conscience populaire. Le mot fit fortune et se propagea, en traînée de poudre, dans le monde entier. Le terme fut adopté par toutes les langues de la terre. Et lorsque les armées hitlériennes commencèrent leur marche conquérante à travers les pays de l'Europe, et qu'il apparut que - de même que lors de la marche des armées du général Franco sur Madrid elles y étaient accueillies, soutenues, aidées, par des éléments amis qui y étaient déjà, c'est tout naturellement et, d'ailleurs avec le bon droit qu'on a appliqué à ces 'amis de l'ennemi' l'appellation : 'cinquième colonne' ", Ibid., pp.8-9.

14. Ibid., p.14.

15. Ibid., p.15.

16. Ibid., p.17. 
17. Ibid., p.130.

18. Lois qui visaient à prévenir contre les idées jugées trop subversives.

19. Jeanne Chase, «L'élaboration de l'immigrant américain ", Annales, 49è année 4, juillet-août 1994.

20. «Returns of Enemy Aliens » War of 1812 Papers, 1775-1812, Naval Records Collections of the Office of Naval Records and Library Recorded Group 45, National Archives, Washington D.C., édition de microforme no.1637, cité par J. Chase op. cit..

21. Voir Sophie Body-Gendrot, Les Etats-Unis et leurs immigrés, Les études de la documentation française, 1991, p.11.

22. Voir la note $\mathrm{n}^{\circ} 11$

23. Pour une analyse historique détaillée voir Jeanne Chase, op. cit..

24. Nous empruntons ce terme à Michael Rogin, Les démons de l'Amérique; Essai sur l'histoire politique des Etats-Unis, Paris, Le Seuil, 1998.

25. Annick Foucrier, "Immigration et tensions raciales aux Etats-Unis. La Californie : un laboratoire " in Catherine Collomp (dir), Amérique sans frontière, Presses de l'Université de Vincennes, 1995.

26. Georg Simmel, The Sociology of Georg Simmel, New York, The Free Press, 1964.

27. NS : Immigration and Naturalization Service, organisme fédéral qui est placé sous la tutelle du ministre de la justice et qui s'occupe des questions de naturalisation et d'immigration.

28. Le nativisme est un courant anti-immigration développé depuis le XIXe siècle. Combinant des thèses protectionnistes et parfois racistes, il propose de préserver les valeurs anglo-saxonnes et européennes, de recentrer l'identité américaine sur une identité blanche et européenne de souche, ainsi que de restreindre, voire arrêter, l'immigration.

29. FAIR : Federation for the American Immigration Reform, organisation anti-immigrée très influente dans le sud-ouest de la Californie. Elle a été fondée en 1979 par John Tanton qui a été le directeur de l'organisation environnementaliste Sierra Club. S'inspirant des thèses nativistes et culturalistes, elle propose l'arrêt de l'immigration légale en provenance des pays du Sud dont en particulier le Mexique ainsi que le renforcement de la lutte contre l'immigration clandestine. Elle publie de nombreuses études et rapports dont Rethinking Immigration Policy and Ten Steps to Securing America's Borders.

30. Organisme fédéral qui est en charge du contrôle des frontières.

31. Donald Huddle, The Cost of Immigration, Carrying Capacity Network, Washington, 1993.

32. Murray Edelman, op. cit., pp.130-131.

33. Pour une analyse détaillée des arguments rhétoriques déployés pendant la campagne en faveur de la Proposition 187, voir Ayse Ceyhan, « Migrants as a Threat. A Comparative Analysis of Securitarian Rhetoric : The European-Union (France) and the United-States (California)», in Victoria Gray (Ed), Immigration, Control and Citizenship, à paraître.

34. Samuel Huntington, "If not Civilization, What ? Paradigm of the Post-Cold War World», Foreign Affairs, 725, nov-déc 1993, p.190.

35. The U.S. Congres Senate, 1911, pp.690-691.

36. Michael Lind, The Next American Nation. The New Nationalism and the Fourth American Revolution, New York, The Free Press, 1995.

37. Y. Lapid, F. Kratochwill, The Return of Culture and Identity in IR Theory, Boulder, London, Lynne Reinner, 1996.

38. David Campbell, « Violent PerformancesIdentity, Sovereignty, Responsability » in Y. Lapid et F. Kratochwill, The return of..., op. cit., pp.163-181.

39. Peter Brimelow, Alien Nation, Common Sense About America's Immigration Disaster, New York, Random House, 1995.

40. Mexican-American Legal Defense and Educational Fund. Il a été fondé en 1968 à Los Angeles. Il est subventionné par la Ford Foundation et les grandes entreprises. 
41. Committies Organized for Public Service, fondée en 1972 est une organisation basée à San Antonio où les deux tiers des habitants est d'origine mexicaine.

42. Voir Didier Bigo, Polices en réseaux, l'expérience européenne, Paris, Presses de Sciences Po, 1996.

INDEX

Mots-clés : discours sécuritaire, ennemi

Index géographique : Etats-Unis 